\title{
ANALYSING RELEVANT INTERACTIONS BY BRIDGING FACEBOOK AND MOODLE
}

\author{
Luciana Oliveira ${ }^{1}$, Alvaro Figueira ${ }^{2}$ \\ ${ }^{1}$ CICE / ISCAP \& Polytechnic of Porto (Portugal) \\ ${ }^{2}$ CRACS / INESC TEC \& University of Porto (Portugal)
}

\begin{abstract}
Social media networks' popularity has been growing in almost every context of daily human interaction. Particularly concerning the education field, organisations and teachers have been continuously recognizing social media as a rich environment with potential to benefit the teachinglearning process, classroom administration and social interactions.
\end{abstract}

However, social media networks have been used as complementary environments to the mandatory adoption of an institutional LMS, leading to the development of fragmented teaching-learning environments, where mutual interchanges are not consolidated nor allow for an explicit academic legitimacy, computation and management. Also, social media networks' integration in education has been viewed as an ad-hoc initiative of some educators, who are prone to incorporate web trends in their pedagogic activity, which are evaluated, most of the times, under the lens of recreational initiates.

Consequently, there is an urgent need to bridge between the consolidated adoption of LMSs and the integration of social media networks in education, not only in terms of technological infrastructure (interface and usability) but also in terms of production and management of its pedagogical outcomes.

With the intent of providing solutions for the above context, in this paper, we discuss the concept of Social Student Relationship Management and present the EduBridge system, its current stage of development and highlight the educational applicability of a thorough set of social network analysis.

Keywords: Social Media Networks, Social SRM, EduBride Social, education, social interactions

\section{INTRODUCTION}

The exponential growth of social media usage and the integration of digital natives in Higher Education Institutions have been posing new challenges to both traditional and technology-mediated learning environments. Nowadays social media plays an important, if not central, role in society, for professional and personal purposes. However, it's important to highlight that in the mind of a digital native, social media is not just a tool; it is a place that is as real and as natural as any real-life world place where formal/informal social interactions happen. Still, formal higher education contexts are still mostly imprisoned in locked up institutional Learning Management Systems, while a new world of social connections grows and develops itself outside schools. One of the main reasons we believe to be persisting in the origin of this matter is the absence of a suitable management, monitoring and analysis tools to legitimize and to efficiently manage the educational and social relationship with students in social networks.

In this paper we discuss the growing relevance of the Social Student Relationship Management (Social SRM) concept and present the EduBridge Social system, which aims at connecting the most commonly used LMS, Moodle, and the most popular social network, Facebook. We describe the motivation underlying this need and its benefits. We, then, focus on the Facebook social network and on its groups as a privileged environment for discussions, much in the same line as forums, however, with much more flexibility. We stress the importance of social media groups, reporting on new findings from a case study where Moodle and Facebook are combined for educational purposes.

\section{SOCIAL... STUDENT RELATIONSHIP MANAGEMENT}

As in any sustaining relationship, a defining feature is the quality of the communication. The educational environment is no exception, since the quality of the communication and the relationships developed in a learning system significantly impact on its efficiency and, when combined, the state-ofthe-art information technologies, mainly social media, offer unlimited resources for the communication's streamlining and diversification of formal and informal learning $[1,2]$. 
Social SRM comprises a set of communication domains that aim to foster student integration and avoid disengagement or dropout, namely the set of relationship and interactions developed between teachers and students, in social media, in the domains of: teaching-learning communication, classroom administration and students' social integration. It contributes to setting a welcoming school climate prone to success and completion, which has been defined as "...the quality and character of school life experiences and reflects norms, goals, values interpersonal relationships, teaching, learning and leadership practices, and organizational structures" [3]. For instance, in a blended learning environment, social media provides two-way exchanges between the classroom and the virtual interaction environment in the relationship building process - both environments being interchangeably reinforced.

Social SRM is composed of three main dimensions:

(1) The domain of teaching-learning communication, in which communication serves as a vehicle for reaching educational goals. It encompasses formal educational communication, which is sustained by a curriculum and pedagogic model with clear indications of contents, methods, requirements and assessment, and the informal spontaneous educational communication, which is not institutionalized, methodical and structured.

(2) The classroom administration related communication domain, which regulates learning tasks, schedules and procedures, since social media has proven to be an excellent tool for processes clarification and celerity, student responsiveness and time economy.

(3) Finally, the student social integration communication dimension, aimed at fostering the formation of the sense of belonging and ultimately the development of a learning community. It consists of the purest form of social interaction, relying heavily on the establishment of meaningful conversations that allow for the development of social and emotional bonds. From the teachers' point of view, and as a community builder/manager, it requires an engaging, motivational, cooperative and personal/informal communication approach that is favoured in social media environments.

\section{THE EDUBRIDGE SYSTEM}

Social media has been boosting educators to drive formal learning contexts into social networks aiming for the development of learning communities that allow the collaborative exploration and reflection of ideas, in a cooperative and supportive atmosphere [4]. Besides offering participants the potential to benefit from a ready source of peer support it also provides the necessary emotional support [5] to allow for communities' social inclusion.

However, in order to efficiently manage and assess social students' relationships, teachers need suitable monitoring and analysis tools/applications. There is also the broader need to institutionally and educationally legitimize the use of social networks, since some teachers refuse to adopt concurrent non-institutional systems, along with the school's LMS. This sometimes lays on top of deep cultural issues and on the organization's proneness to adopt/adjust to new technologies.

Given this scenery, we believe to be of the utmost importance to bridge between the well-established LMS and the widely used social networks (ex. Facebook), taking advantage of pre-acquired habits, knowledge and sense of control, which is currently offered to teachers. We believe that providing the current LMS with insights and features that allow teachers to manage and perceive the dynamics of students' interactions on social media, to manage them and to make educational sense of them, may, in fact, facilitate and potentiate the inclusion of social networks on the best service of education.

\subsection{System's Architecture}

The proposed system is based on a set of Moodle blocks that share a communications engine and a database stored locally. The system's architecture is based on the model-view-control pattern, implemented through five modules. In this case, the 'view' is produced by each module. However, the 'control' is hierarchically spread through the top level-module down to the leaves modules. This organization is depicted in Fig. 1. As a top layer we have the communications module (googleoauther2) which establishes a connection between Moodle and Facebook through the Facebook's authentication mechanism, using the user's credentials. Then, the control is passed to the module fbgroups to retrieve the groups owned by the user. This module is also responsible for creating lists of users belonging to the groups and to set some counters for usage statistics. The module fbcomments retrieves all the posts and comments that were posted in timeline of the selected 
group. Finally, the modules fbstats and fbtotalstats present the local statistics, and global statistics, about the selected group or all the considered groups, respectively.

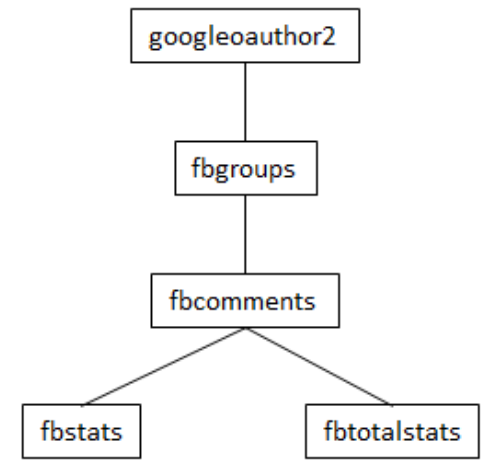

Fig. 1: The system's five modules

\subsection{System's Interface}

The system's interface is composed of a four-set Moodle blocks which users, particularly teachers, can display and manage in their Moodle Dashboard (known as "My home" up to Moodle's 2.8 version). This aims at framing the system in the most recent Moodle's philosophy for managing the teacher's educational workspace.

The four blocks are designated as: "Facebook Groups", "Facebook Posts \& Comments", "Facebooks Statistics" and "Total Statistics".

The block's behaviour is interdependent, thought the information displayed on all four blocks is adjusted to users' interaction with the block "Facebook Groups". The "Facebook Groups" block retrieves the list of groups that the authenticated user manages on Facebook. This means that all educational and personal groups are displayed and accessible through Moodle's interface. This is also the main block among the four-set, since it allows teachers to arrange the information and views to be generated.

In order to provide teacher with tools to (1) separate personal from educational groups and (2) arrange and match the groups to their courses on Moodle a set of dropdown matching boxes were incorporated in this block. This allows teachers to create as many Facebook groups as needed and match them with Moodle courses, for a broader or narrower analysis.

At the current stage of development, the EduBridge Social system offers teachers with the ability to:

- Login into Moodle with Facebook credentials;

- Access Facebook group feed in Moodle "My home" (Module "Facebook posts and comments");

In block "Facebook Groups":

- Access the full list of groups the user manages on Facebook;

- Match the groups to existing Moodle courses (one or more Facebook groups per Moodle course);

In block "Group statistics":

- Access statistical data concerning the group selected in block "Facebook Groups", such as: total number of posts, total number of comments, median of comments per post, visualise the most recent post, retrieve the most popular post in the group, access the list of users who posted in the group (and the corresponding amount of posts and likes), access the list of users who commented on posts (with total number of replies and likes);

In block "Facebook total statistics":

- Access the aggregated statistics for all Facebook groups assigned to a specific Moodle course: total number of posts within the groups, as well as total amount of likes, comments, replies and comments' likes; the group with recent updates, users who posted the most and who interacted the most within all the course groups.

In section four, we report on a range of social networks analysis which believe to be of great added value for the educational context and which we are incorporating on EduBridge Social. Data used in 
these analysis is the same as used primarily for the initial development of the system. New results are presented and we discuss their relevance on social network analysis (SNA) in education.

\section{SOCIAL NETWORK ANALYSIS USING THE SYSTEM}

Theoretically, the SNA is a process of investigating social structures through the use of network and graph theories. During this analysis the networked structures in terms of nodes (actors, people, or things within the network) and ties or edges (relationships or interactions) that connect them are characterized.

\subsection{The Case Study}

In this section we present evidence on previous research conducted on the use of Facebook groups as an extension of the classroom teacher-student relationship, into a more dynamic and informal environment. The aim of this case study was to investigate and assess the three components of the educational dimension of Social SRM mentioned in section two.

The study was carried out during two semesters, in three different subjects from the first, second and third year from two different courses. The courses were held in traditional classroom, with Moodle support. A Facebook group was created for each class and the students were invited to join. Though participation was not mandatory, all of the students having a Facebook profile joined the groups, except for 3 students who didn't own a Facebook profile and didn't wish to own one. Among the three classes there were 99 students (grouped in 52, 34 and 13 participants) and one teacher interacting in three separate Facebook groups. Facebook groups were introduced to students as a complimentary support platform in the first class of the semester and not as a replacement for the institutional LMS, as Wang [6] proposes.

We must stress that the focus lies on the communicative / dialogical nature of the interactions happening inside the learning communities, mainly because there was no particular objective assigned to participants when these communities were formed. Students were invited to join a "support page" where anything could happen, in order to assure that dialogue and relationships were spontaneous and self-motivated, as already defended by some authors [7].

All the groups' interactions were retrieved from Facebook, using the available API. In this process, the following fields were collected on each groups feed: post id, post author, post message, post type and corresponding \#comments and \#likes.

\subsection{Learning analytics and visualisation}

As the methodology for getting analytical insights is the same for every Facebook group, for the sake of simplicity and space, we restrict the analysis to a single Facebook group.

\subsubsection{Types of interactions}

From the original 162 posts we were able to retrieve the associated data, namely the comments for the posts and the number of likes. We also distinguish the post type, which in case of Facebook may be one of 4 types: link, photo(s), status change and video. We recall that we retrieved the posts for the first semester of 2013, which comprehend posts/comments between September 2013 and January 2014. The results are presented in Table 1.

Table 1. Analytics of the retrieved posts.

\begin{tabular}{|l|r|r|r|}
\cline { 2 - 4 } \multicolumn{1}{c|}{} & \multicolumn{1}{c|}{ Posts } & Comments & \multicolumn{1}{c|}{ Likes } \\
\hline Number of & 162 & 772 & 727 \\
\hline Post type: & \multicolumn{3}{l}{} \\
\hline Link & 30 & 80 & 113 \\
\hline Photo & 35 & 263 & 205 \\
\hline Status & 90 & 413 & 372 \\
\hline Video & 7 & 16 & 37 \\
\hline
\end{tabular}

These results provide awareness of the total amount of interactions in a social network during a certain amount of time. It also offers perspectives on the type of content which is more prone to 
generate higher participation and engagement for that community in particular and which are the most common types of interactions. For this case study, "comments" and "likes" are typically four times higher than the amount of messages posted in the group. "Posts" consist mainly of "status" messages (text messages without any media addition) and these are the type of content to gather the higher number of "comments" and "likes" which were collected. However, post type "photo" is the most prone to generate higher levels of interaction: whereas "status" messages generate an average of 4 "comments" and "likes", "photo" type posts are able to collect, in average, 8 "comments" and 6 "likes".

This analysis can provide teachers and students with relevant information regarding the type of post they should choose when looking to increase their interaction with other group members. The analysis is also relevant for teachers looking to develop comparisons among several groups he/she manages, to outline a specific group profile and to transfer knowledge, as group manager, from one group into another.

\subsubsection{Frequency of interactions}

We then analysed the posting frequency using the metric "posts per day". As shown on Fig. 2 this number varies between 1 and 7 . Analysing the posting frequency/intensity in the course of the semester allows us to identify peaks of intensity of communication / interaction occurring in the group.

Particularly in the case study presented, it is possible to identify 4 distinct moments: one in early October, one in late November, one in December and one in mid, late January. The visualisation of the intensity of interactions in the group provides teachers with the detection of the most intense working periods along the semester. It could also reveal how students organize class projects and which could be the most productive periods. In this case, students were assigned a four-month collaborative project and an individual project. On early October, higher interactions are related to deciding the themes and teams for the collaborative project. Peaks on late November and throughout December are related to intra and inter team discussions and teacher solicitations for the development of the projects. The peaks detected on the first and third week of December indicate the higher stages of project development closer to its due date.

Depending on the results obtained in formal assessment and the quality of these peaks of interactions, teachers might feel necessary to make adjustments to course schedules and to try to anticipate or delay certain peaks aiming at improve the overall performance of students and of the learning process.

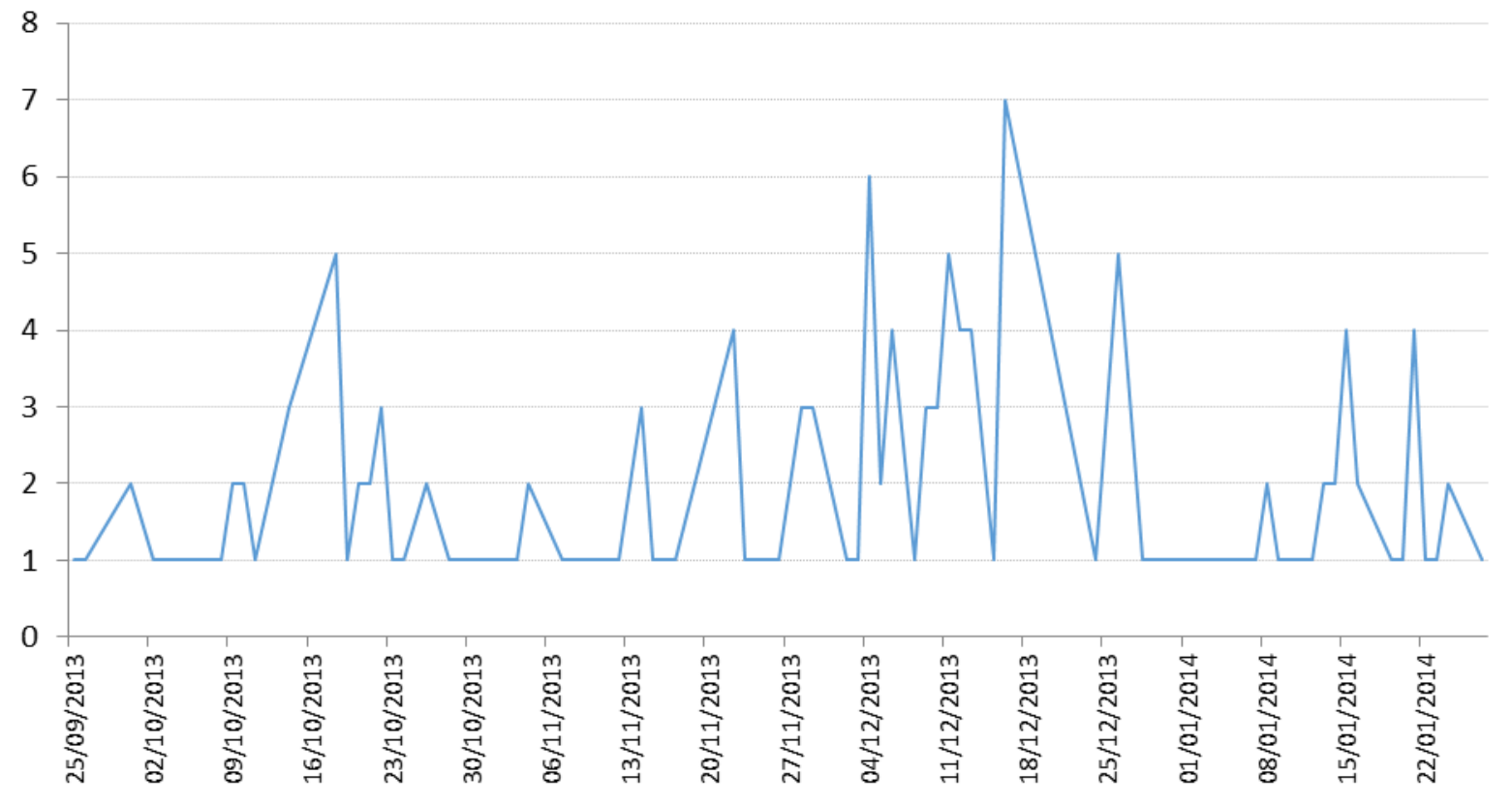

Fig. 2: Facebook group posting frequency 
On the Moodle side, the frequency of interaction with class resources and activities sheds light on the course schedule as well, as illustrated in

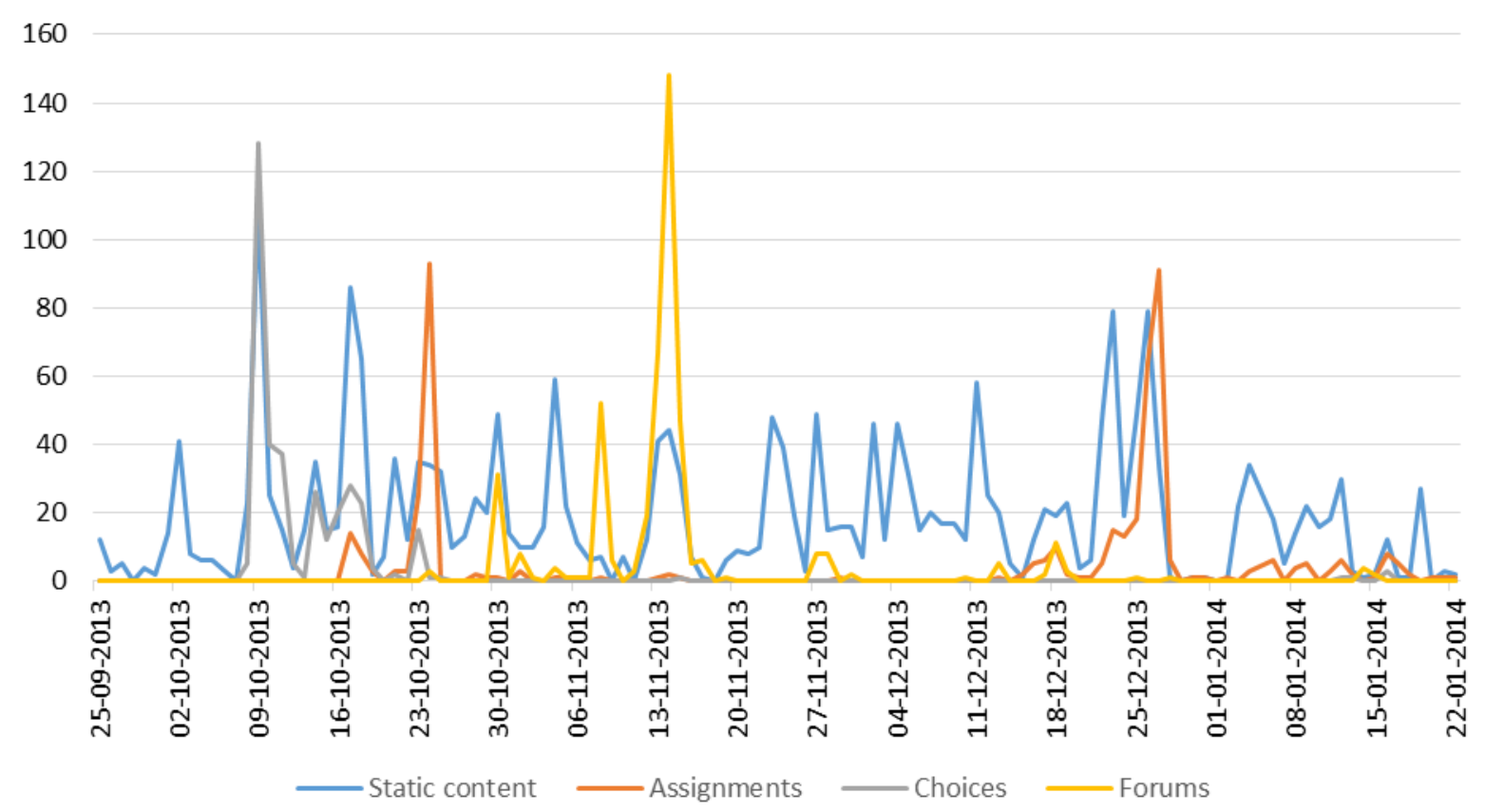

Fig. 3.

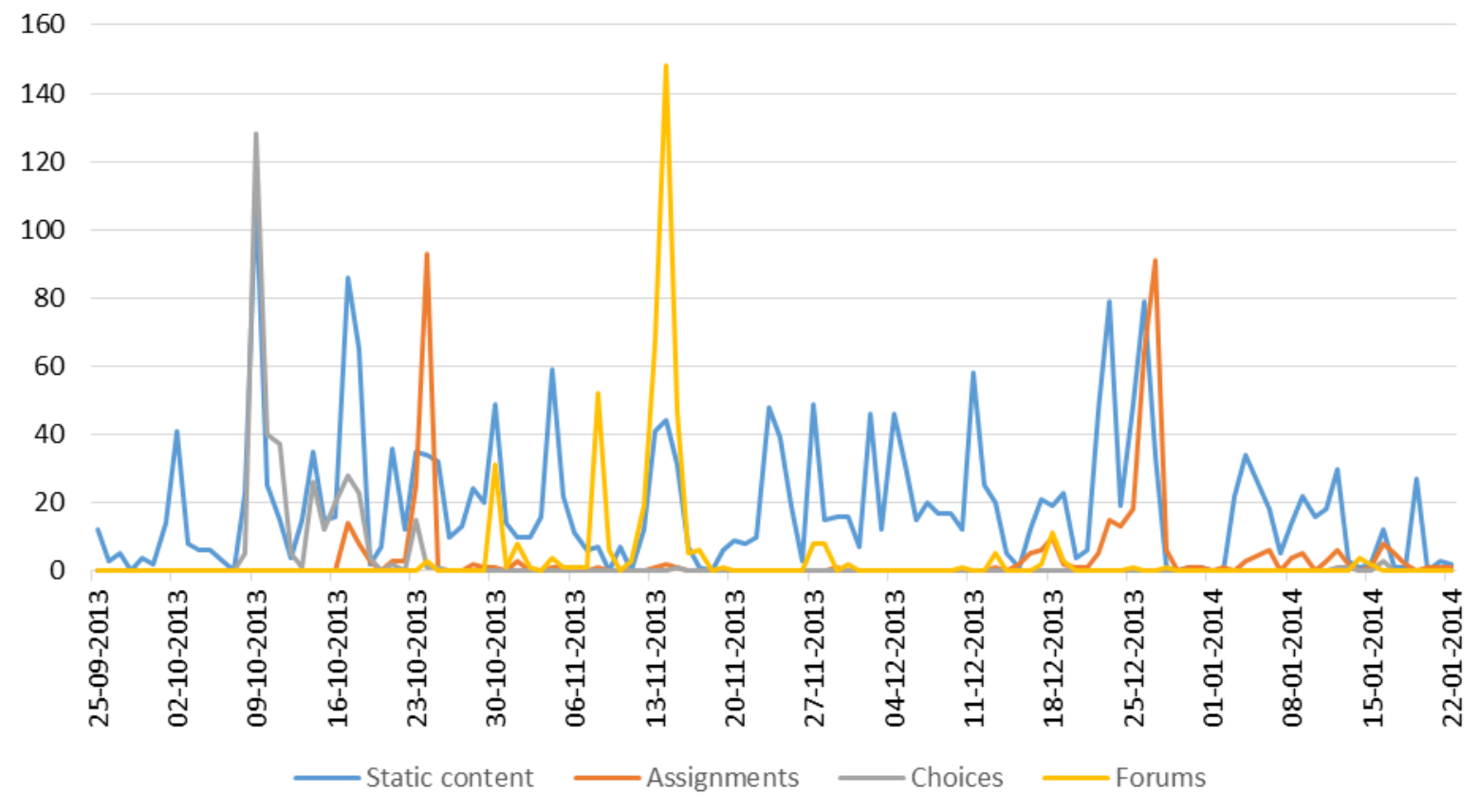

Fig. 3: Frequency of interaction of Moodle resources / activities

We are only displaying the most relevant Moodle actions for this course: access to static course contents; view and submission of assignments; use of the "Choice" tool for groups' formation and use of Moodle forums to discuss assignments. Overlapping Figures 3 and 4 allows us to obtain a clear image of the complementarity relation that has been established between Moodle and Facebook and on how interactions (with resources and with others) are mutually reinforced.

This combination of results from both environments is of the highest importance for the assessment of the overall conduction of a course, particularly for the teacher. It also allows the teacher to evaluate the potential offered by each of the environments and to better plan for future integrations. When using several educational environments teachers should be aware of the added value these bring into the educational context and should also be able to report on and to account for it. 


\subsubsection{Daily interactions' periods}

One of the main advantages of Social Media Networks, particularly Facebook, is its ability and easiness to fit into student's daily digital environment. Educators' ability to take advantage of this scenario may return higher student involvement in the learning process and higher proximity between the academic and personal life for students, allowing for bigger interchanges. Social media is also a privileged environment for news/content sharing. Whether it consists on specialized/technical or generic content, real-life events, case studies or curiosities, its incorporation in educational groups is a one or two clicks process. Therefore, educational groups on Social Media benefit from the speed and diversity of content permanently emerging on the network.

As reported by other studies [8], best times to post on Facebook are early in the morning, between work and dinner hours and between dinner and bedtime. Data from our case study (as illustrated in Fig. 4) consolidates these indicators, reinforcing the period between around 4 p.m. as the most active in group interactions. As mentioned above, this educational context is benefiting from Social Media constant usage habits.

Analysing the most active and fruitful time slots when looking to foster interactions is a valuable insight for teachers, whether it is knowledge acquired from previous group analysis or is related the group under analysis. Though every group may tend to develop a very intrinsic interaction profile, which may be more or less permeable to internal or external factors, the collection of insights and knowledge gathered by the teacher are of high instrumental value for the development of learning communities'

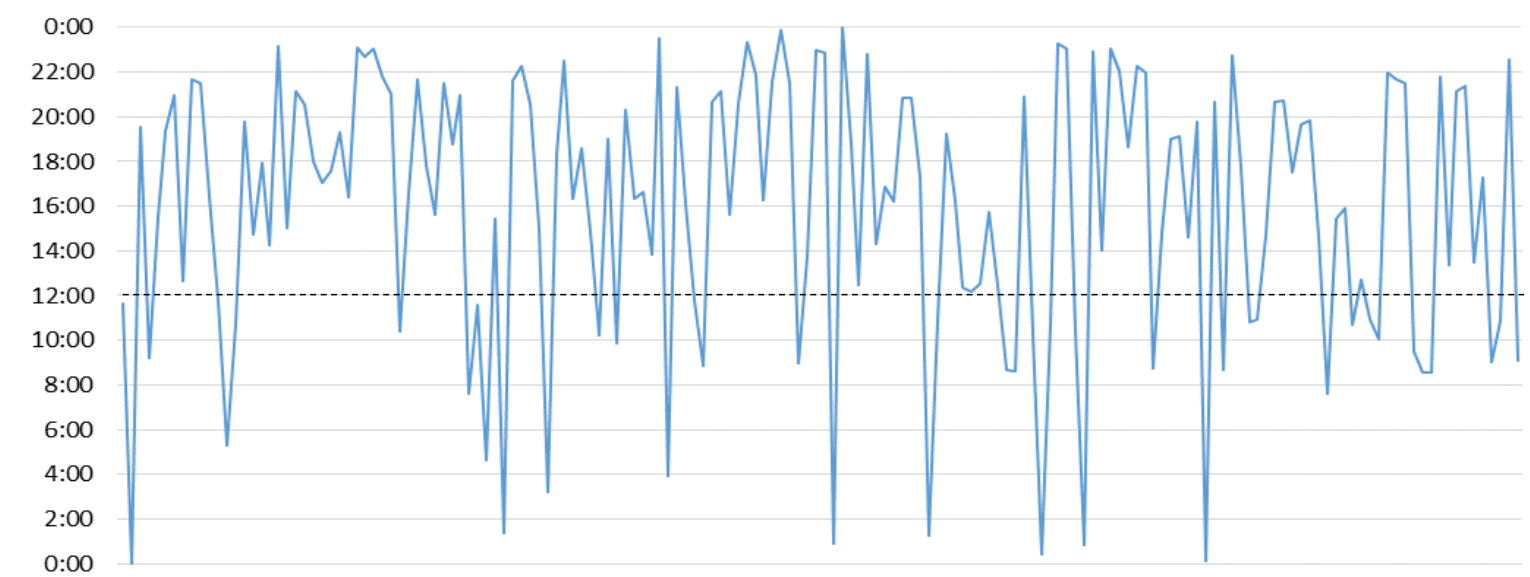

management skills.

Fig. 4: Interactions per hour of the day

\subsubsection{Visualising interactions}

Traces of activity left by social media users can facilitate perceptions on individual behaviour, social relationships, and community efficiency [9]. Tools and processes to analyse social traces are essential for enabling educators to study and nurture meaningful and sustainable social interactions occurring in learning communities.

In the case study under analysis we have a Facebook group with 42 participants, which correspond to the 42 nodes of the social graph represented in Fig. 5. There are 556 established connections (in the form of a 'comment-to' or 'reply-to') between group members, but only 51 are unique. This is why some connections are thicker than others, to depict a repeated connection. There are 150 self-loops, which mean that some group participants do comment, or reply, to their own posts, or comments. 


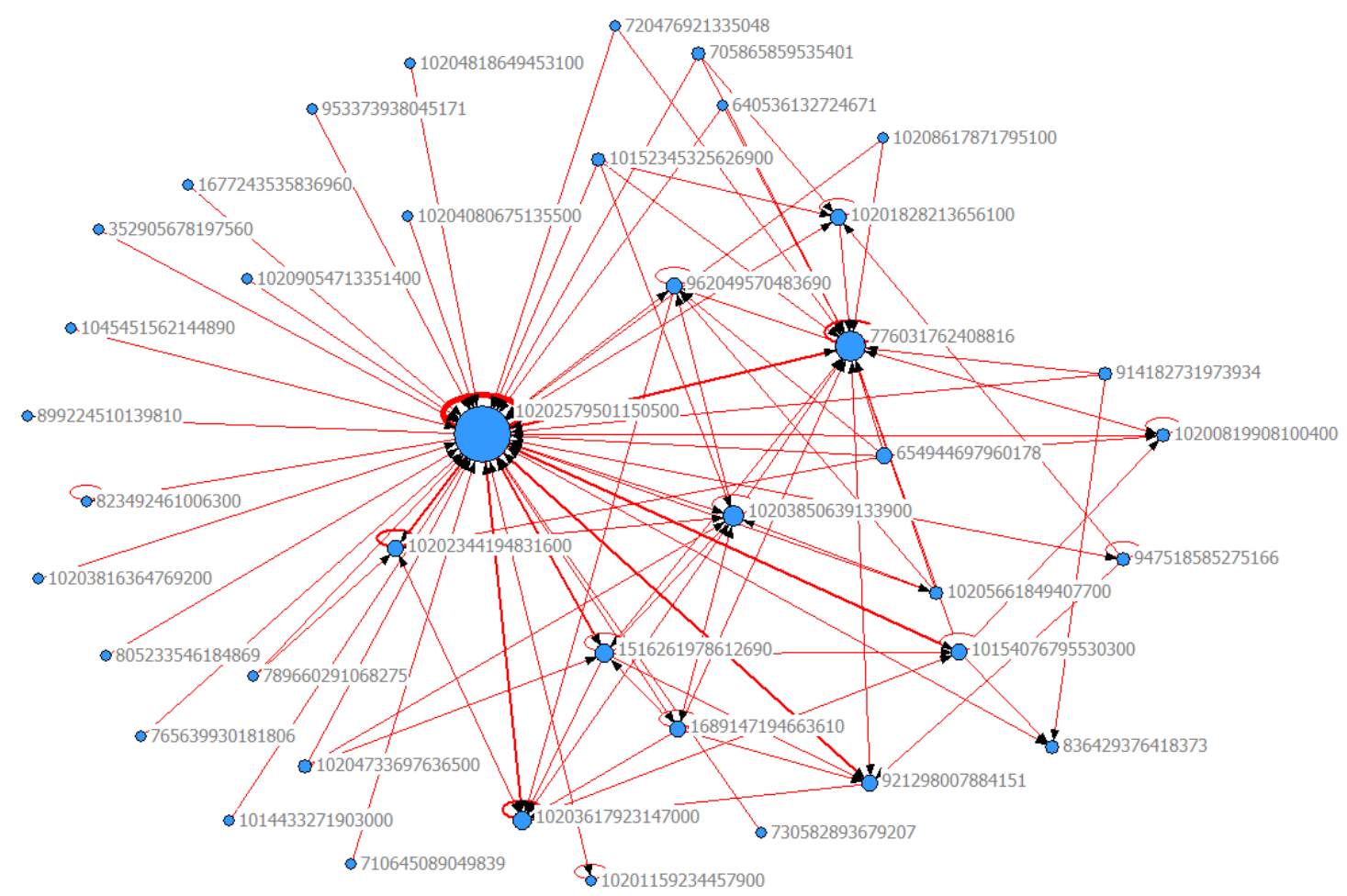

Fig. 5. The social graph

Identities on the social graph have been anonymised, and converted to masked Facebook profile IDs, in order to assure confidentiality. In our representation of the network we adhere to the format proposed in [10]. As it can be seen in Fig. 5, some nodes are bigger than others. This happens because we set the size of each node to be proportional to its out-degree (not that the graph is directed). Therefore, the biggest the node size, the largest is its out-degree. This metric is a simple way to find out the most participative people in the group. We can also easily find which node ("102...50500" - the teacher) bridges all the others, although node "776...08816" (student) is also important in the network. Another conclusion that we can deduct from the graphic is that only a minority of nodes (apart from the teacher) are connected to other nodes. I.e., students do not frequently engage in each other conversations or are not very solicited. However, there are about 15 exceptions ( 15 nodes) to this rule.

The previous analysis also leads us to identify a graph density of about $6 \%$. This value levels to a poorly connected network, which is a characteristic of Ego-Networks or of high-centralized networks. On the other hand, the maximum geodesic distance is 3 , while the average geodesic distance reduces to 1.9 , which means that we can, on average, reach any other node in less than two hops. Therefore, despite not being a dense network, it is well connected (because of the high centralization index).

Clearly, there are nodes with more connections and connected with denser parts of the network. Those nodes (students) allow the information to propagate fast, and make the network more responsive to posts. Mapping this situation to the academic world and students, this means that these students are indispensable to have success in communicating to the whole class. As a side note, it is curious to note that all these subgraphs include self-loops, which means that these students are willing to create answers in their own posts or comments, fostering discussion around topics that they brought to the group.

Educational insights on graph interaction analysis provide teachers with knowledge on which type of community has developed or is being developed. Clearly, this group and its interactions are very teacher centric. There are a few students generating interactions among peers but the bridge element is the teacher. Many considerations could be built on this topic: weather the interactions should be teacher or student centred, which are not our main purpose. He can however stress that knowledge on this aspect is both relevant for teachers and for students, namely: to identify the type of community, its main intervenient, the origin of interactions and how oneself relates to the others in that group. 


\section{CONCLUSIONS}

Social Network Analysis insights are an extremely valuable resource for pedagogical optimization. They also foster the teachers' continuous self-development and self-learning process, adding awareness as closing the gap between teacher and students' perceptions of the teaching-learning process and dynamics.

One of the challenges faced by teachers when using social media as component of the educational infrastructure lies on the difficulty to compute, assess and legitimize the social interactions that are dynamically generated inside a community. Being able to turn these interactions into tangible outputs that can be incorporated, for instance, in formal assessment, might provide social media with the certified educational status it has been lacking. Naturally, this question raises several analogous other, which we do not intend to discuss in this paper. What we wish to stress here is that educational providers have validated and approved LMSs as an essential component of technology mediated education, regardless of which use teachers make of them, but have been failing at acknowledging and validating social media networks as institutional educational value added environments.

We observe that, despite the existence of some meritorious, but only isolated, efforts to integrate social media networks into the learning process in general, and even into online learning environments in particular, there is still a lack of a clear rationale for that integration. Moreover, there are no available tools to provide teachers with substantial learning analytics to manage that integration, particularly when addressing issues that go beyond simple SNA metrics. We believe we are at the Frontier of that educational rationale, and that EduBridge will converge to a global first step into a solid Social Student Relationship Management tool.

\section{ACKNOWLEDGMENTS}

This work is financed by the ERDF - European Regional Development Fund through the COMPETE Programme (operational programme for competitiveness) and by National Funds through the FCT Fundação para a Ciência e a Tecnologia (Portuguese Foundation for Science and Technology) within project «Reminds/ UTAP-ICDT/EEI-CTP/0022/2014».

\section{REFERENCES}

1. Crammer, K. and Y. Singer, On the algorithmic implementation of multiclass kernel-based vector machines. The Journal of Machine Learning Research, 2002. 2: p. 265-292.

2. Oliveira, L., Social Student Relationship Management in Higher Education: extending educational and organisational communication into Social Media. 2015.

3. Bryant, V.C., et al., School as haven: Transforming school environments into welcoming learning communities. Children and Youth Services Review, 2013. 35(5): p. 848-855.

4. Palloff, R.M. and K. Pratt, Online learning communities in perspective. Online learning communities, 2007: p. 3-15.

5. Bruchman, A., Learning, _. in Online Communities. 2006.

6. Wang, Q., et al., Using the Facebook group as a learning management system: An exploratory study. British Journal of Educational Technology, 2012. 43(3): p. 428-438.

7. Deng, L. and N.J. Tavares, From Moodle to Facebook: Exploring students' motivation and experiences in online communities. Computers \& Education, 2013. 68: p. 167-176.

8. Cormier, D. When Is the Best Time to Post on Facebook? 2013; Available from: http://www.socialmediatoday.com/content/when-best-time-post-facebook.

9. Hansen, D.L., et al. Do You Know the Way to SNA?: A Process Model for Analyzing and Visualizing Social Media Network Data. in Social Informatics (Sociallnformatics), 2012 International Conference on. 2012. IEEE.

10. Silva, A. and Á. Figueira. Depicting online interactions in learning communities. in Global Engineering Education Conference (EDUCON), 2012 IEEE. 2012. IEEE. 